\title{
Comportamento de oviposição da mosca-negra-dos-citros Aleurocanthus woglumi (Hemiptera: Aleyrodidae) em plantas cítricas
}

\author{
Oviposition pattern of citrus blackfly Aleurocanthus woglumi \\ (Hemiptera: Aleyrodidae) on citrus plants
}

\author{
Adalton Raga ${ }^{1}$, João Filipe Muniz Basilli ${ }^{1}$, Daniel Zaniboni Soares ${ }^{1}$
}

\begin{abstract}
RESUMO
O objetivo do presente estudo foi avaliar o comportamento de oviposição de Aleurocanthus woglumi Ashby em plantas cítricas. O estudo foi conduzido em pomar de laranjeira, instalado no município de Artur Nogueira (São Paulo, Brasil). Foram observadas 16.125 posturas da mosca-negra-dos-citros em 256 folhas. A partir da avaliação de 1280 posturas (cinco/folha) obteve-se o mínimo de 8 , o máximo de 50 e a média de 28,42 ovos por postura. Aproximadamente $83 \%$ das posturas tinham entre 16 e 41 ovos. O número de posturas no quadrante Sul foi significativamente superior ao quadrante Oeste e semelhante aos demais quadrantes. Não houve diferença estatística entre os quadrantes quanto ao número de ovos por postura.
\end{abstract}

Palavras-chave: insecta, praga dos citros, ecologia, comportamento, oviposição.

\begin{abstract}
The objective of this study was to evaluate the behavior of Aleurocanthus woglumi Ashby oviposition in citrus plants. The study was conducted in an orange orchard installed in the municipality of Artur Nogueira (State of São Paulo, Brazil). We observed 16,125 egg masses from 256 fully expanded leaves. From 1,280 egg masses of A. woglumi (five per leaf) we obtained a minimum of 8 , a maximum of 50 and an average of 28.42 eggs per mass. Approximately $83 \%$ of the egg masses had from 16 to 41 eggs. The egg masses in the south quadrant were significantly more numerous than in the west quadrant and similar to the remaining quadrants. No differences were detected among the average number of eggs per mass in each quadrat.
\end{abstract}

Key words: insect, citrus pests, ecology, behavior, oviposition.

Plantas cítricas são hospedeiras de um grande número de pragas em todo o mundo. Ebeling (1959) listou aproximadamente 875 espécies de insetos e ácaros em citros e Talhouk (1975) considerou 144 espécies como pragas. Espécies de Aleyrodidae (Hemiptera) ampla distribuição geograficamente e apresentam um grande número de plantas hospedeiras silvestres e ornamentais (Cassino e Nascimento, 1999).

A família Aleyrodidae, com 161 gêneros e 1556 espécies descritas, é dividida em três Subfamílias: Aleyrodinae, Aleurodicinae e Udamoselinae (Martin e Mound, 2007). A Subfamília Aleurodicinae contém espécies endêmicas, consideradas mais primitivas, distribuídas principalmente na América do Sul e América Central.
Originária do sudeste da Àsia (Hart et al. 1978), a mosca-negra-dos-citros Aleurocanthus woglumi Ashby (Hemiptera: Aleyrodidae) foi registrada pela primeira vez no Brasil, em julho de 2001 no estado do Pará (Oliveira et al., 2001) e se disseminou para 20 estados brasileiros (MAPA, 2007; Lemos et al., 2006; Raga e Costa, 2008; Raga et al., 2011). No Estado de São Paulo, a espécie foi detectada pela primeira vez em março de 2008, sendo encontrada em sete municípios na região centro-leste paulista, infestando severamente laranjeiras, tangerineiras e limoeiros em (Raga e Costa, 2008).

Aleurocanthus woglumi pode infestar mais de 300 espécies de plantas em todo o mundo (Nguyen e Hamon, 1993). Os danos diretos são causados por ninfas e adultos, pela sucção contínua de

\footnotetext{
1 Instituto Biológico, Centro Experimental, Rodovia Heitor Penteado km 3, Caixa postal 70, CEP 13001-970, Campinas, SP, Brasil. E-mail: adalton@biologico.sp.gov.br
} 
nutrientes das folhas e conseqüente depauperamento das plantas. Os danos indiretos são oriundos da fumagina, um sintoma decorrente do desenvolvimento de um fungo negro sobre o exsudato das ninfas de A. woglumi em folhas e frutos, o qual dificulta a fotossintese e a respiração das plantas (Raga e Costa 2008).

A mosca-negra-dos-citros tem característica de realizar a postura em forma espiral e de forma agrupada (Dowell \& Cherry 1981), variando de 35 a 50 ovos por postura, sendo estes depositados na superfície inferior das folhas (Nguyen e Hamon 1993).

As dificuldades para o manejo da mosca-negra-dos-citros são devidas, entre outras limitações, ao desconhecimento sobre a ecologia da praga em nossas condições. Assim, o objetivo do presente estudo foi caracterizar em pomar comercial de citros, o comportamento de oviposição de $A$. woglumi.

Utilizou-se um pomar de laranja 'Westin' (Citrus sinensis L. Osbeck) enxertadas sobre Limão Cravo (Citrus limonia), com seis anos de idade, plantado no espaçamento de $7,0 \mathrm{~m} \times 4,0 \mathrm{~m}$ e instalado no Sítio São Bento, município de Artur Nogueira, SP

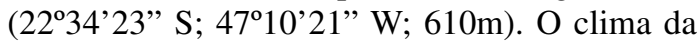
região é caracterizado como clima CWA, segundo a classificação de Köppen (1948). No período de 02/06 a 03/07/2008, na região do pomar estudado, a temperatura média variou entre $16,1^{\circ}$ e $18,2^{\circ} \mathrm{C}$ e a precipitação pluviométrica acumulada foi de $36,9 \mathrm{~mm}$. O pomar estava livre da aplicação de inseticidas por aproximadamente 20 dias antes da coleta de folhas.

A amostragem foi realizada em plantas em bom estado de enfolhamento. No dia 03/07/2008 foram coletadas quatro folhas maduras por quadrante em dezesseis plantas ao acaso, distanciadas de pelo menos 12 metros entre si, totalizando 256 folhas. As folhas foram individualizadas por quadrante, sendo acondicionadas em sacos de papel e transportadas em caixas de isopor para o Laboratório de Entomologia Econômica do Instituto Biológico, em Campinas, SP, Brasil.

Para avaliar o comportamento de oviposição de A. woglumi foi utilizado um microscópio esteroscópico, com aumento de 40 vezes, através do qual observou-se o número de posturas recentes por folha, com características íntegras, ou seja, com ou sem eclosão recente de larvas. O número de ovos/ postura/folha foi avaliado em cinco posturas recentes escolhidas ao acaso. Foi adotado o delineamento inteiramente casualizado, sendo considerada cada planta uma repetição. Os dados foram transformados em raiz quadrada de $(\mathrm{x}+1)$ e submetidos aos testes F e Tukey $(\mathrm{p} \leq 0,05)$.

Foi contado um total de 16.125 posturas obtendo-se em média, 63,0 posturas por folha. Em cada quadrante, o número médio de posturas por folha variou entre 51,02 e 70,30. O número de posturas no quadrante Sul foi significativamente superior ao quadrante Oeste, sendo este semelhante aos demais quadrantes (Tabela 1). Em plantas cítricas na época seca, Medeiros et al. (2009) observaram no Estado do Maranhão, maior número de posturas de $A$. woglumi nos quadrantes norte e sul na época seca, enquanto que na época chuvosa não houve diferença entre os quadrantes, em vista da menor infestação da praga. Assim, acredita-se que as diferenças de infestação observadas entre os quadrantes são devidas às características de maior infestação da praga na época de menor precipitação pluviométrica. Os dados diferem daqueles obtidos por Cherry \& Fitzpatrick (1979), que não observaram diferenças de infestação de $A$. woglumi entre os quadrantes de plantas cítricas.

A partir de 1.280 posturas da mosca-negra-dos-citros, obteve-se o mínimo de oito e o máximo de 50 ovos, sendo a média de 28,42 ovos por postura. Não houve diferença estatística entre os quadrantes quanto ao número de ovos por postura (Tabela 1). Mesmo submetidas à alta infestação natural de $A$. woglumi, não foi detectada postura na face superior das folhas, embora fossem observadas áreas comuns entre posturas. Aproximadamente $83 \%$ das posturas tinham entre 16 e 41 ovos (Figura 1). Pena et al. (2008), obtiveram em laboratório a média de 27,6 ovos por postura em folhas de C. sinensis, padrão

Tabela 1. Número médio de posturas por folha ( \pm erro padrão) e de ovos de Aleurocanthus woglumi em cinco posturas ( \pm erro padrão), obtidos em laranja doce.

Artur Nogueira, SP, Brasil. Julho de 2008.

\begin{tabular}{lcc}
\hline Quadrante & $\begin{array}{c}\mathrm{N}^{\mathrm{o}} \text { médio de posturas/ } \\
\text { folha } \pm \mathrm{EP}\end{array}$ & $\begin{array}{c}\mathrm{N}^{\mathrm{o}} \text { médio de ovos/cinco } \\
\text { posturas } \pm \mathrm{EP}\end{array}$ \\
\hline Norte & $70,30 \pm 6,08^{1} \mathrm{ab}$ & $139,39 \pm 4,91 \mathrm{a}$ \\
Sul & $70,98 \pm 5,61 \mathrm{a}$ & $141,19 \pm 5,36 \mathrm{a}$ \\
Leste & $59,66 \pm 4,60 \mathrm{ab}$ & $139,03 \pm 4,27 \mathrm{a}$ \\
Oeste & $51,02 \pm 4,89 \mathrm{~b}$ & $149,02 \pm 4,55 \mathrm{a}$ \\
\hline
\end{tabular}

1 Médias seguidas pela mesma letra na coluna não diferem entre sipelo teste de Tukey $(\mathrm{P} \leq 0,05)$. 


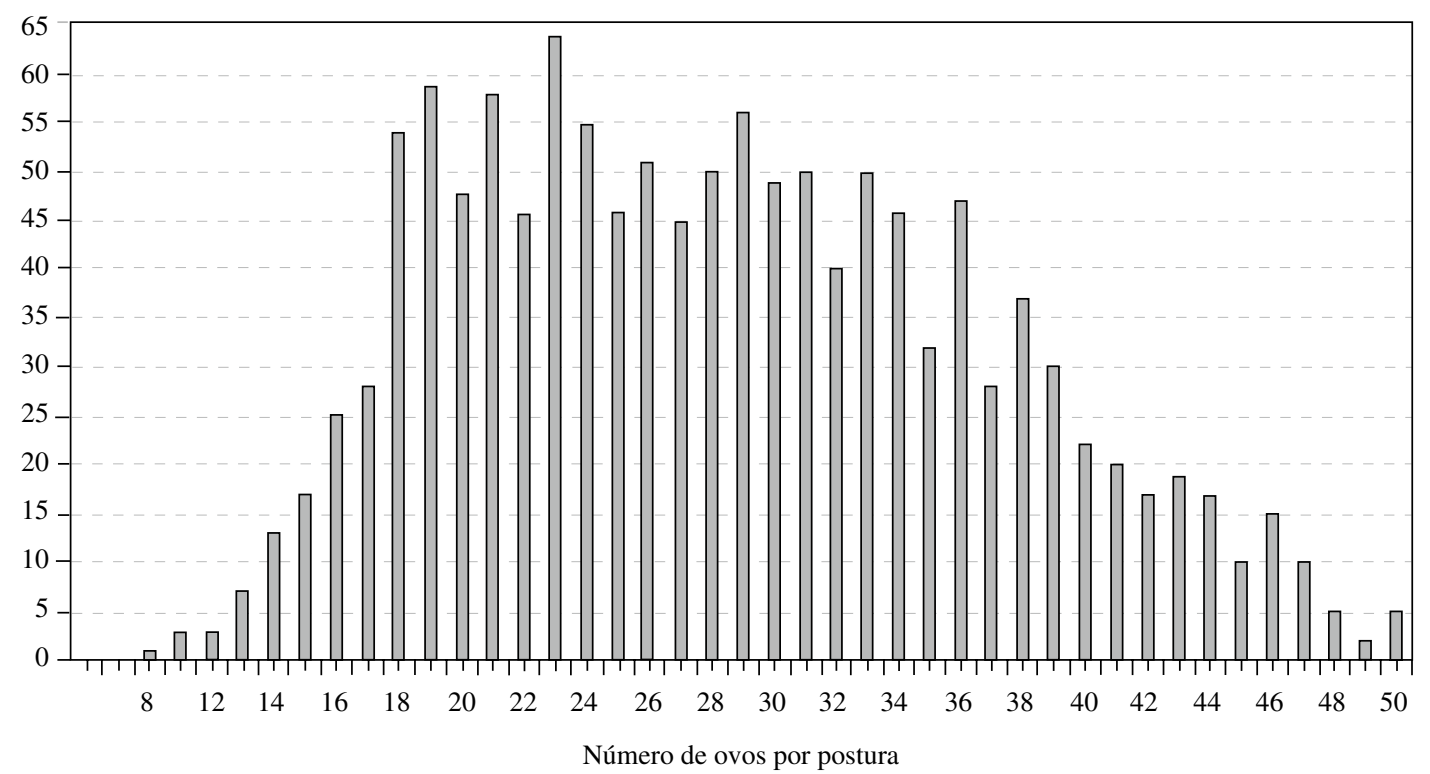

Figura 1. Frequência do número de ovos por postura de Aleurocanthus woglumi observada em folhas de laranja doce (Citrus sinensis) em Artur Nogueira, SP, Brasil. Julho de 2008.

muito semelhante ao encontrado no presente trabalho, embora este tenha sido conduzido em condições naturais.

A mosca-negra-dos-citros apresenta posturas agregadas, sendo desconhecida a possível vantagem desse comportamento (Dowell e Cherry 1981).
O comportamento de oviposição, aliado com altos níveis de infestação observados no presente experimento demonstram o elevado potencial biótico de A. woglumi em citros, tornando recomendável a adoção do manejo integrado da praga para alcançar o controle populacional da praga.

\section{Literatura Citada}

Cassino, P.C.R.; Nascimento, F.N.

1999 Aleirodídeos (Homoptera: Aleyrodidae) em plantas cítricas no Brasil: distribuição e identificação. Anais da Sociedade Entomológica do Brasil 28: 75-82.

Cherry, R.H.; Fitzpatrick, G.

1979 Intra-tree dispersion of citrus blackfly. Environmental Entomology 8: 997-999.

Dowell, R.V.; Cherry, R.H.

1981 Detection of, and sampling procedures for, the citrus blackfly in urban southern Florida. Researches on Population Ecology 23: 19-26.

Ebeling, W.

1959 Subtropical Fruit Pests. University of California, Division of Agricultural Sciences, $436 \mathrm{p}$.

Hart, W.G.; Selhime, A.; Harlan, D.P.; Ingle, S.J.; Sanchez, R.M.; Rhode, R.H.; Garcia, C.A.; Caballero, J.; Garcia, R.L. 1978 The introduction and establishment of parasites of citrus blackfly, Aleurocanthus woglumi in Florida (Hem.: Aleyrodidae). Entomophaga, 23 (4): 361-366.

Köppen,W.

1948 Climatologia: com un estudio de lós climas de la tierra. México: Fundo de Cultura Econômica, 478 p.
Nguyen, R.; Hamon, A.B.

1993 Citrus blackfly, Aleurocanthus woglumi Ashby (Homoptera: Aleyrodidae). Gainesville Florida Department of Agriculture \& Consumer Service - Division of Plant Industry, 3 p. Circular $\mathrm{N}^{\circ} 360$.

Lemos, R.N.S.; Silva, G.S.; Araújo, J.R.G.; Chagas, E,F.; Moreira, A.A.; Soares, A.T.M.

2006 Ocorrência de Aleurocanthus woglumi Ashby (Hemiptera: Aleyrodidae) no Maranhão. Neotropical Entomology 35: 558-559.

Martin, J.H.; Mound, L.A.

2007 An annotated check list of the world's whiteflies (Insecta: Hemiptera: Aleyrodidae). Zootaxa 1492: 1-84.

Medeiros, F.R.; Lemos, R.N.S.; Ottati, A.L.T.; Araújo, J.R.G.;

Machado, K.K.G.; Rodrigues, A.A.C.

2009 Dinâmica populacional da mosca-negra-dos-citros Aleurocanthus woglumi Ashby (Hemiptera: Aleyrodidae) em Citrus spp. no município de São Luís - MA. Revista Brasileira de Fruticultura 31: 1016-1021.

Mapa - Ministério da Agricultura, Pecuária e Abastecimento 2007 Instrução Normativa 52. Disponível em <www.extranet. agricultura.gov.br/sislegis-consulta/18316. Acesso em: 10 set 2010 . 
Oliveira, M.R.V.; Silvia, C.C.A; Navia, D.

2001 Mosca negra dos citros Aleurocanthus woglumi: alerta quarentenário. Brasília, Ministério da Agricultura, Pecuária e Abastecimento, $12 \mathrm{p}$.

Pena, M.R., Silva, N.M., Vendramim, J.D., Lourenção, A.L., Haddad, M.

2008 Biologia da Mosca-Negra-dos-Citros, Aleurocanthus woglumi Ashby (Hemiptera: Aleyrodidae), em Três Plantas Hospedeiras. Neotropical Entomology 38: 254-261.

Raga, A.; Costa, V.A.

2008 Mosca negra dos citros. São Paulo: Instituto Biológico, 9 p. (Documento Técnico 001). Disponível em: <http://www. biologico.sp.gov.br/docs/dt/mosca_negra.pdf>. Acesso em: 16 jul. 2010.

Raga, A.; Marsaro junior, A.L.; Racca Filho, F.; Costa, V.A. 2011 Novos Registros de Espécies de Aleyrodidae (Hemiptera) no Estado de Roraima, Brasil. Arquivos do Instituto Biológico 78: 439-441.

Talhouk, A.S.

1975 Citrus pests throughout the world, distribution and importance. In: Citrus: Ciba-Geigy Agrochemicals Technical Monograph 4, pp. 21-28. 